\title{
Zwischen fordern und überfordern
}

\author{
Drei Jahre SGB II - eine Zwischenbilanz
}

\author{
Karen Peters
}

Seit Anfang 2005 ist das Zweite Buch Sozialgesetzbuch (SGB II) in Kraft, in dem die frühere Arbeitslosenhilfe und die Sozialhilfe für erwerbsfähige Personen zusammengeführt wurden. Der Deutsche Verein für öffentliche und private Fürsorge zieht im Jahre Vier nach Hartz IV eine Zwischenbilanz.

Kaum eine Reform hat die Gemüter mehr bewegt und ähnliche öffentliche Debatten ausgelöst als das im Volksmund unter »Hartz IV « bekannt gewordene »Grundsicherung für Arbeitsuchende«. Bereits durch 25 Änderungsgesetze ist das noch junge Werk geändert, fortentwickelt, verschärft, klargestellt, präzisiert, an neue Erkenntnisse und Entwicklungen angeglichen worden. Die durch das SGB II eingeführte Rechtsfigur der »Bedarfsgemeinschaft « wurde in mehreren Wellen erweitert, der Kanon der Sanktionen bei fehlender Mitwirkung im fast halbjährlichen Turnus weiter verschärft und der Reigen der Eingliederungsmaßnahmen immer weiter verfeinert, auf neue Personengruppen zugeschnitten. Sollte der neueste Entwurf zur Reform der Instrumente wie geplant in Kraft treten, wird alleine \16 SGB II auf $\mathbb{\int} 16$ bis $16 \mathrm{~h}$ angewachsen sein und viele Seiten des Gesetzbuches beanspruchen.

Als wäre die Praxis mit der Umsetzung immer neuer Regelungen im Gesetz und einer ständig hinterherhinkenden Software nicht ausreichend beschäftigt, steht nun auch noch die organisatorische Durchführung der Aufgaben zur Disposi-

Karen Peters ist Leiterin des Arbeitsfeldes »Grundlagen sozialer Sicherung, Sozialhilfe und soziale Leistungssysteme « beim Deutschen Verein für öffentliche und private Fürsorge e. V. Dort ist sie seit mehreren Jahren mit Fragen in Zusammenhang mit der Umsetzung des SGB II befasst. Internet http://www.deutscher-verein.de tion. Im Dezember 2007 hat das Bundesverfassungsgericht die Aufgabenwahrnehmung durch die Arbeitsgemeinschaften - ein wenig geglückter Kompromiss im Gesetzgebungsverfahren - für unvereinbar mit dem Grundgesetz erklärt. Unter dem Titel »Drei Jahre SGB II: Erfahrungen, Auswirkungen, Schlussfolgerungen « hat der Deutsche Verein für öffentliche und private Fürsorge ein Themenheft der Fachzeitschrift »Archiv für Wissenschaft und Praxis der sozialen Arbeit « herausgebracht, in der Vertreter aller beteiligten Organisationen zu Wort kommen und eine, oft kritische, Zwischenbilanz ziehen:

- Wolfgang Spellbrink, Richter am Bundessozialgericht, setzt sich darin in seinem Beitrag mit der Frage auseinander, ob die Regelleistung des SGB II ausreicht, um den Lebensunterhalt zu sichern. Kritik ist nicht nur an der Bemessung immer wieder laut geworden, sondern auch an der fehlenden Individualisierungsmöglichkeit im SGB II.

- Die Bedarfsgemeinschaft, so Christian Armborst, Ministerialrat im Niedersächsischen Sozialministerium in seinem Aufsatz, sei eines der wackeligen Standbeine, auf denen das " eigentümliche "Wesen des SGB II stehe und das »so manchen Ästheten unter den Dogmatikern schaudern lasse «. In der Tat hat der Gesetzgeber der Rechtsprechung, der Wissenschaft und vor allem der Praxis mit der Einführung der Bedarfsgemeinschaft eine harte Nuss zu knacken gegeben, die in ihrer Umsetzung viele Fragen aufwirft.

- Mit den Kosten der Unterkunft, dem großen Kostenblock im SGB II, setzt sich Uwe Berlit, Richter am Bundesverwaltungsgericht, auseinander. Von massenhaften "Zwangsumzügen « war gleich zu Beginn in der Presse die Rede; es werde viel zu großzügig Geld für teure Wohnungen ausgegeben, wetterte jüngst der Bundesrechnungshof. Die Hilfeempfänger sehen das anders und beschäftigen die Sozialgerichte mit Rechtsmitteln gegen die Ablehnung ihrer Unterkunftskosten; jedes fünfte Klageverfahren betreffe die Kosten der Unterkunft.

- Die Reform stand unter dem Schlagwort »Fordern und Fördern«: Vorrangiges Ziel war und ist es, erwerbsfähige Hilfebedürftige wieder in Arbeit zu bringen. Dazu sollten alte Instrumente verbessert werden, neue geschaffen und vor allem die Kompetenzen der beiden Partner Bundesagentur für Arbeit und Kommunen zusammengeführt werden. Die Zumutbarkeit für die Annahme von Tätigkeiten wurde verschärft, der Abschluss einer Eingliederungsvereinbarung mit dem Hilfebedürftigen (zumindest in der Theorie) obligatorisch. Ob diese Maßnahmen frischen Wind auf den Arbeitsmarkt gebracht haben, oder doch nur ein laues Lüftchen, damit setzen sich Susanne Koch und Ulrich Walwei vom Institut für Arbeitsmarkt- und Berufsforschung auseinander.

- Martina Musati und Wilhelm Falk von der Bundesagentur für Arbeit stellen Ergebnisse, Probleme und Perspektiven der aktiven Arbeitsmarktförderung dar. Während bei der Qualität des Fallmanagements und der Vernetzung der Träger nach ihrer Ansicht noch vieles zu verbessern ist, sind Erfolge beispielsweise bei der Reduzierung der Jugendarbeitslosigkeit und bei der Nutzung der differenzierten Instrumente des SGB III festzustellen.

- Mit der starken Fokussierung auf den Aspekt des »Forderns « im SGB II, der häufig auch zu einer Über «forderung « der Hilfeempfänger führen kann, setzt sich Helga Spindler, Professorin an der Universität Duisburg/Essen, kritisch auseinander. Die strengen Voraussetzungen an die Zumutbarkeit und die gerade für junge Menschen einschneidenden Sanktionsdrohungen verschieben ihrer Ansicht nach auf dem Ar- 
beitsmarkt das Kräfteverhältnis zulasten der Arbeitnehmer. Das gelte nicht zuletzt auch für die sogenannten EinEuro-Jobs.

- Gerade diese könnten aber für viele Hilfebedürftige eine echte Chance sein,
Chancen als an der gerechten Verteilung von Gütern gemessen.

- Die Entscheidung des Bundesverfassungsgerichts vom Dezember 2007 und ihre möglichen Auswirkungen schließlich finden nicht nur Eingang

\section{"Gerechtigkeit wird heute anhand gleicher Chancen, nicht an der gerechten Verteilung von Gütern \\ gemessen «}

wieder Fuß im Arbeitsleben zu fassen, meint Thomas Inden, Geschäftsführer der allerhand $g G m b H$ zu Köln, der über die weitgehend positiven Erfahrungen der Caritas an 17 Modellstandorten berichtet, in denen unter Wahrung von ausreichenden Qualitätsanforderungen zusätzliche Beschäftigungsmöglichkeiten geschaffen wurden.

- Mit der Rolle der sozialen Dienstleistungen der Kommunen in der Grundsicherung für Arbeitsuchende und den Schnittstellen zu anderen kommunalen Handlungsfeldern beschäftigt sich der Beitrag von Regina Offer vom Deutschen Städtetag. Die Zusammenarbeit sei nicht zuletzt wegen der unterschiedlichen Blickwinkel und Erwartungen der Hilfeleistenden in der Praxis häufig noch verbesserungsfähig.

- Zu den gesellschaftspolitischen Hintergründen, die zu der Entscheidung geführt haben, mit der Grundsicherung für Arbeitsuchende ein Leistungsgesetz zu schaffen, das sich ausdrücklich an Erwerbsfähige richtet, schreibt Stephan Rixen, Professor am Institut für Sozialpolitik und Sozialer Dienste an der Universität Kassel: »In einer solchen Welt ist im Grunde kein Raum für einen Arbeitsbegriff, der nicht um die Erwerbsarbeit kreist und kein Raum für Modelle, die (...) nicht die Erwerbsarbeit zur Basis der Lebensunterhaltssicherung erheben.«

- Heinz-Jürgen Dahme und Norbert Wohlfahrt beleuchten den Wandel der Gerechtigkeitsdebatte im Lichte des aktivierenden Sozialstaates, der immer mehr auf die Eigenverantwortung und die private Vorsorge setzt. Gerechtigkeit wird heute mehr anhand gleicher in jeden der Beiträge, sondern sind auch Gegenstand einer eigenen Abhandlung von Markus Mempel vom Deutschen Landkreistag, der sich mit möglichen Handlungsoptionen auseinandersetzt.

Der Deutsche Verein möchte mit der Zusammenschau der unterschiedlichen Sichtweisen neue Erkenntnisse und Aspekte in die aktuelle Diskussion einbringen, die sicherlich auch im vierten Jahr »nach Hartz IV « nicht abbrechen wird.

Drei Jahre SGB II: Erfahrungen, Auswirkungen, Schlussfolgerungen. Archiv für Wissenschaft und Praxis der sozialen Arbeit 1/2008. Preis mit Mitglieder des Deutschen Vereins für öffentliche und private Fürsorge: 10,70 Euro (Bestellung perE-Mail thomas.ulber@cvk.de); für Nichtmitglieder: 14,50 Euro (Bestellung perE-Mailnfo@lambertus.de).

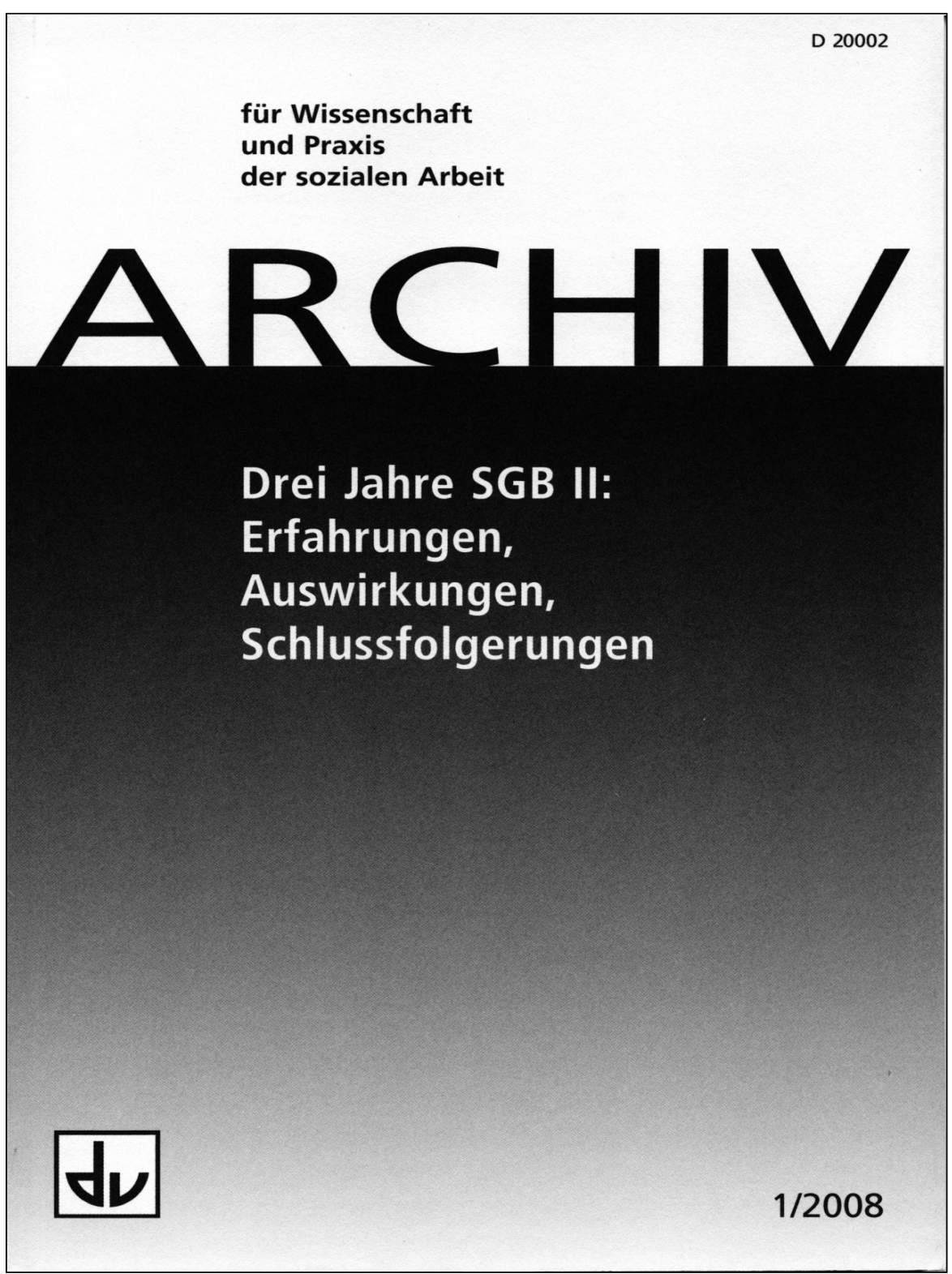

